\title{
USING DISCRETE EVENT SIMULATION TO EXAMINE MARINE TRAINING AT THE MARINE CORPS COMMUNICATION-ELECTRONICS SCHOOL
}

\author{
Jon Davenport \\ Charles Neu \\ William Smith \\ Susan Heath \\ Naval Postgraduate School \\ 555 Dyer Road \\ Monterey, C.A. 93943, U.S.A.
}

\begin{abstract}
This paper presents a discrete-event simulation model used to explore various possibilities for improving the training continuum at the Marine Corps CommunicationElectronics School. The goal of the analysis is to reduce the average waiting time experienced by Marines as they wait for their formal training to commence. Results show that the implementation of even the least beneficial of these improvements yields a 37 percent reduction in waiting time. The best single change yields an 82 percent reduction. This translates into a 30 day reduction in average waiting time per Marine. If all improvements were implemented, a reduction of 88 percent could be achieved, bringing the average waiting time per Marine down to less than 5 days.
\end{abstract}

\section{INTRODUCTION}

This paper presents a discrete-event simulation model used to explore various possibilities for improving the training continuum at the Marine Corps CommunicationElectronics School (MCCES). MCCES, located in 29 Palms, CA, is a formal learning center that provides Marine Corps Operating Forces with entry-level and careerprogression training for enlisted Marines in the communications occupational field.

One of the entry-level training courses taught at MCCES is the Tactical Data Network Operators Course (TDNOC), Military Occupational Specialty (MOS) 0656. Upon completion of the course, 0656 Marines graduate with the basic requisite knowledge, skills and abilities to install, configure, operate and maintain networking systems to include switches, routers, and various transmission media. The 0656 Marine is a crucial component to the Marine Corps' ability to fight the nation's battles; therefore, any time a Marine spends waiting for training is time lost for the Operating Forces.

The purpose of this paper is to analyze the flow of 0656 Marines through the training continuum in order to assess the efficiency of the current system with regard to the time Marines spend waiting for a class to begin. In addition, the paper explores the effects of several potential improvements to the system.

MCCES currently trains approximately 420 MOS 0656 Marines per year. This data comes from the Training Input Plan (TIP), which is a document developed by higher headquarters that estimates the total number of Marines to pass through the system per trimester per fiscal year. The TIP becomes the source document used for planning and scheduling purposes at MCCES. One of the underlying goals of MCCES is to reduce the cycle time of Marines by moving them through the system as quickly and efficiently as possible within each course's particular constraints. There are limited resources available to train the 0656 Marines that cycle through TDNOC each year. One MCCES representative stated that analyzing these resources objectively and quantitatively has yet to be done. Consequently, there may be inefficiencies that, once identified, could be "Leaned" out of the system or optimized to in order to reduce the time that Marines spend in the system.

By building and analyzing a discrete event simulation model of the 0656 training continuum, the authors were able to obtain an average baseline of the time the 0656 Marines spend awaiting training which was 36 days. In addition, several potential improvements were tested using the Process Analyzer Function (PAN) in Arena. Results show that the implementation of the least beneficial of these improvements yields a 37 percent reduction in waiting time. If all improvements were implemented, a reduction of 88 percent could be achieved, bringing the 
average waiting time per Marine down to less than five days.

The remainder of the paper is organized as follows: The current state of the training continuum and the model we constructed are described in Section 2. Section 3 details the potential improvements that were investigated. The simulation model is presented in Section 4. The experimental design and the results are presented in Section 5. The conclusions and future work are discussed in Section 6.

\section{CURRENT SYSTEM}

Working off of available data, in fiscal year (FY) 2006 there were 46 weeks in which MOS 0656 Marine students arrived at MCCES, which is roughly once every seven days excluding holidays. (A FY always runs from October 1 to September 30.) Upon arrival, the students spend three days in an indoctrination course before being transferred to the Marines Awaiting Training (MAT) Platoon where they wait until a scheduled class begins. When a scheduled class start-date arrives, the class can commence provided that the minimum number of students per class criteria is met. If there is an insufficient number of Marines to begin a class, the class is cancelled. If there are more Marines than the maximum capacity of the class, only the maximum number will begin the scheduled class. The remaining Marines must then wait for the next scheduled class to start. Marines are processed on a firstin-first-out (FIFO) basis. Each class consists of a lecture component and a laboratory component. Upon completion of the course, Marines graduate and depart the school. The basic process flow is depicted in Figure 1.

Under standard operating procedures, the 0656 course requires 41 training days, of which the first 15 are spent in a lecture hall and the remaining 26 are spent in the laboratory. Training days are exclusive of weekends and holidays.

By directive, the minimum class size is set at 15 . The maximum class size is determined by the available training aids per laboratory. Each training aid supports two Marines and MCCES currently has 10 training aids available per laboratory. However, historical trends on equipment readiness have caused MCCES to plan for a 10 percent failure rate in each lab. As a result, MCCES generally allocates nine operational training aids per laboratory for a total of 18 Marines per class. Currently two lecture halls and four laboratories are available for the 0656 course.

Class scheduling has generally been calculated by using the TIP data. The projected annual arrivals from the TIP are divided by the maximum class size to derive the total number of classes required per FY. Once the total number of required classes has been determined, class start-dates are spread fairly evenly throughout the year. It is important to note that the arrival rate of 0656 Marines is not evenly distributed throughout the year resulting in large Marines Awaiting Training (MAT) queues. Although 14 billets exist for instructors, the current staffing level is 10 instructors.

To model this training system, the authors constructed a discrete-event simulation using Arena 10.0. Marines were scheduled to arrive in batches on 46 Wednesdays over the course of a year, avoiding holidays. Historical arrival data was entered into Arena's Input Analyzer to determine the best distribution for generating the random number of Marines arriving on a given arrival day. This best fit distribution was: $-0.5+34 *$ $\operatorname{BETA}(1.04,2.62)$. This variation in the number of arriving Marines is the only source of variability in the system, since the processing times for the Indoctrination Course and the two phases of the 0656 course are all deterministic.

The instructors, lecture halls, and laboratories were all modeled as resources. In order to prevent training from taking place on non working days, the instructors were scheduled to not work on all weekends and holidays. It is important to note policy dictates that each class requires two instructors.

In order to better model the ongoing training system, we modeled a 364 day warm-up period. In addition, at the beginning of the warm-up period, we began the system with two classes in progress and twenty Marines in the MAT queue. Statistics for the average time Marines spent in the MAT queue were collected for the 365 days following the warm-up period. In order to achieve a $95 \%$ confidence interval of approximately $+/$ - one percent we ran the model for 1000 replications. This configuration of the model formed the base scenario.

\section{POTENTIAL SYSTEM IMPROVEMENTS}

This research examined several possible scenarios concentrating on changes that could be made to the system that would reduce the time Marines spent waiting for training. Some of these changes would require high-level approval for implementation and others could be implemented by MCCES immediately. For the analysis in this paper, focus was placed on items that could be changed by the local commander and did not require any capital investment. Therefore, this investigation included using a compacted training cycle, increasing the number of instructor billets currently filled, varying minimum and maximum class sizes, and changing to on-demand class scheduling.

The possibility of increasing the number of training days per week and number of training hours per day was examined. This option increased daily training to 10 hours per day from eight, and increased the work week to six days per week. This change shortened the course of 


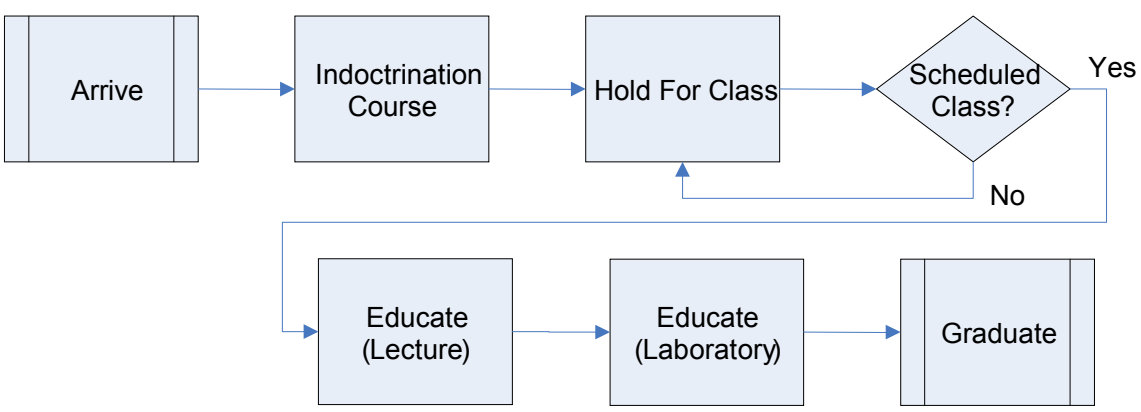

Figure 1: Student flow through MCCES.

instruction to 32 training days and can be implemented in times of crisis when throughput is critical.

Increasing the available number of instructors from 10 to 12 is possible because the school is currently authorized 14 instructors. Keeping all available instructor slots manned at $100 \%$ is unlikely, therefore; consideration was given to increasing the instructor pool to 12 which allows both lecture halls and all four labs to be used simultaneously.

Although the current system dictates a minimum class size of 15 , the authors felt it was necessary to examine the effects of forcing full classes (minimum of 18 students) to be sure there was no benefit to be gained from that change. It is also possible to change the maximum class size by allowing three students to sit at each training aid in the laboratories rather than two. This would increase the maximum class size to 27 students.

Incorporating on-demand scheduling is a broad change which would require a large paradigm shift in order to implement. In on-demand scheduling, a class requires four conditions to be met prior to starting: a minimum number of students, two available instructors, one available lecture hall, and one laboratory that will become available by the time the lecture portion of the course is finished.

\section{SIMULATION MODEL DESCRIPTION}

\subsection{Scheduled Version}

The simulation model was developed using the Arena 10.0 simulation software by Rockwell Automation (www.arenasimulation.com). Two models were created, one to simulate a scheduled operation and one to simulate the on-demand method.

The scheduled-classes model has five main parts: creating Marines in training at time zero (Figure 2); creating Marines already in MAT at time zero (Figure 3); process to read next arrival day (Figure 4); process to read next scheduled class (Figure 5); and the training process (Figure 6).

The logic for creating the Marines in training in both classrooms and labs at time zero is used to simulate the steady-state process of training Marines at MCCES. In addition to initiating the system with Marines already in training and waiting for training, a one-year warm-up period was used. Marines in training are created at the beginning of the simulation in batches simulating formed classes that are progressing through training. Entities are assigned an attribute that is used to track total time in the system. Similarly, Figure 3 contains the logic for creating the Marines in the MAT queue at time zero. Entities are then assigned an attribute that records their arrival time to the system.

Figure 4 shows the section of the model that reads in the arrival days of Marines who arrive on 47 Wednesdays of the calendar year. The 47 days were input into the model logic using historical data provided by MCCES. There is then a delay until the next arrival day is reached. When the arrival day is reached, a random number of Marines are created according to the distribution stated earlier and the arriving Marines are assigned their arrival time and their MOS attribute. Then they go through the 3 day Command Indoctrination training.

The logic for creating the class start dates is dependent on the amount of students awaiting training since there are minimum and maximum class sizes that are limiting factors. Figure 6 shows how a Hold module named MAT is used to simulate students waiting for a signal that a scheduled class is starting. Figure 5 shows how a class start time is read in from the file of actual scheduled class starts and, if there is a sufficient minimum amount of students in the MAT queue, a signal to start a class is sent. Once the signal is sent, all students are released from the MAT queue with a limit up to the maximum students allowed per class in a first in, first out priority, as shown in Figure 6. Setting a class size variable insures that the number of Marines batched into a class exactly equals the number of Marines that were released from the MAT queue to begin training. Any remaining students in the MAT queue must wait there until the same requirements are met to teach another class.

Once the Marines are batched into a class, the class then proceeds through the lecture and lab class sessions. One class uses two instructors for both the lecture and the lab portions and uses one lecture hall and then one lab. 


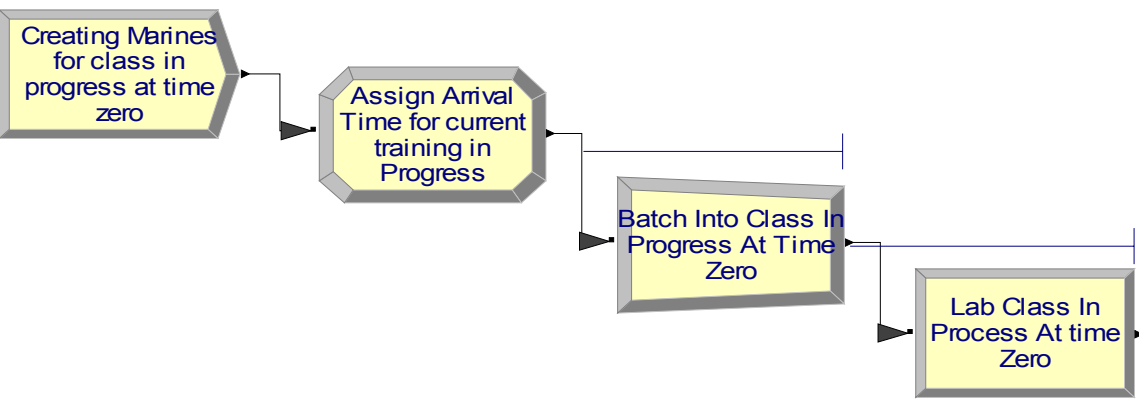

Figure 2: Example of Arena logic for creating Marines in training at time zero.

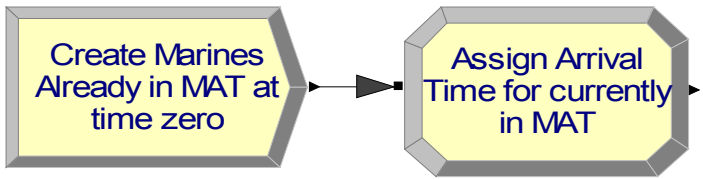

Figure 3: Example of Arena logic for setting up Marines in the MAT queue at time zero.

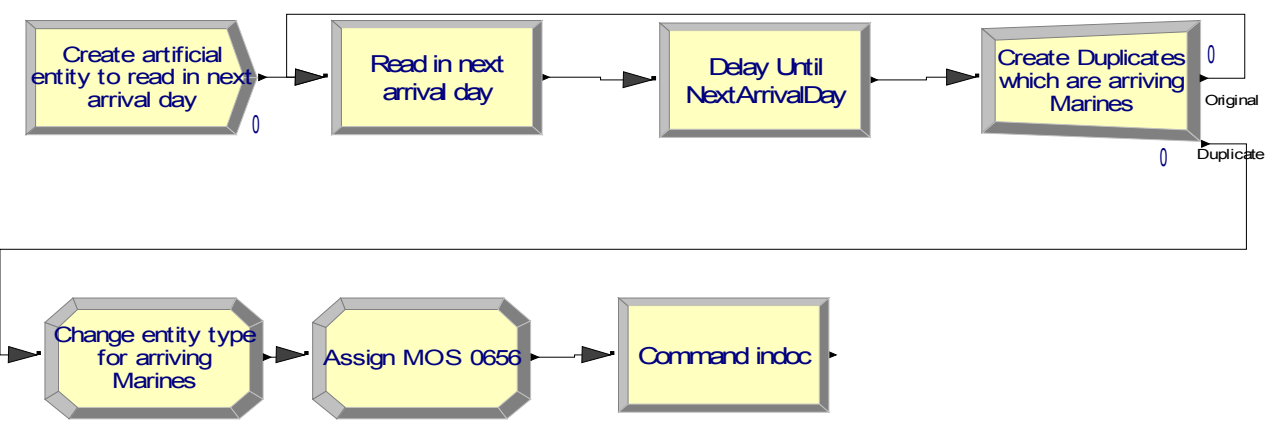

Figure 4: Example of Arena logic for simulating arrival of Marines and their Command Indoctrination training.

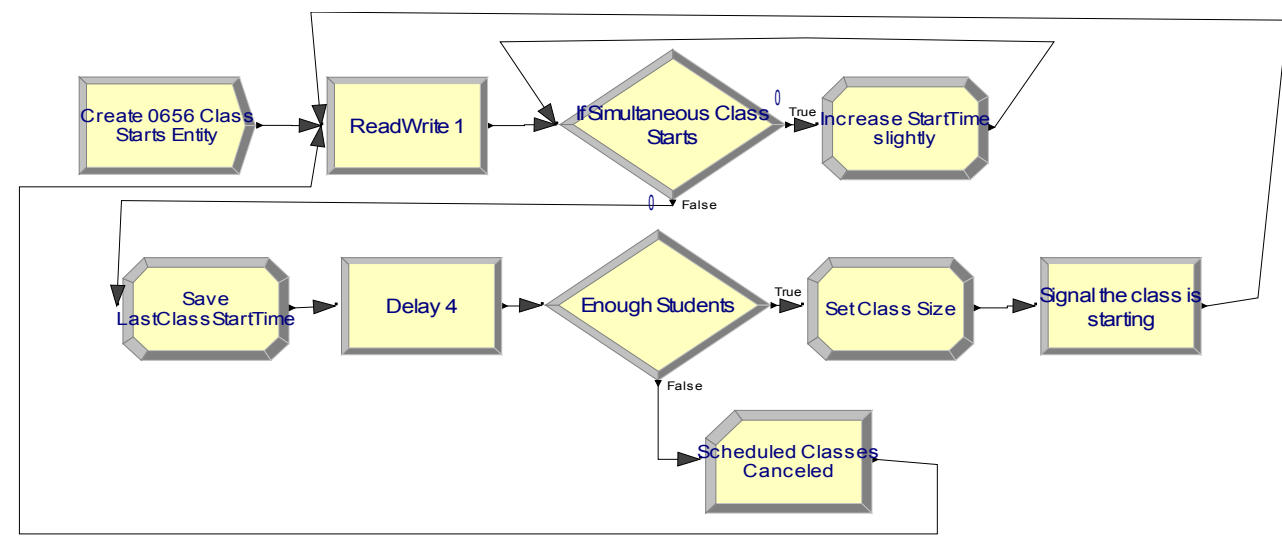

Figure 5: Example of Arena logic for creating scheduled classes. 

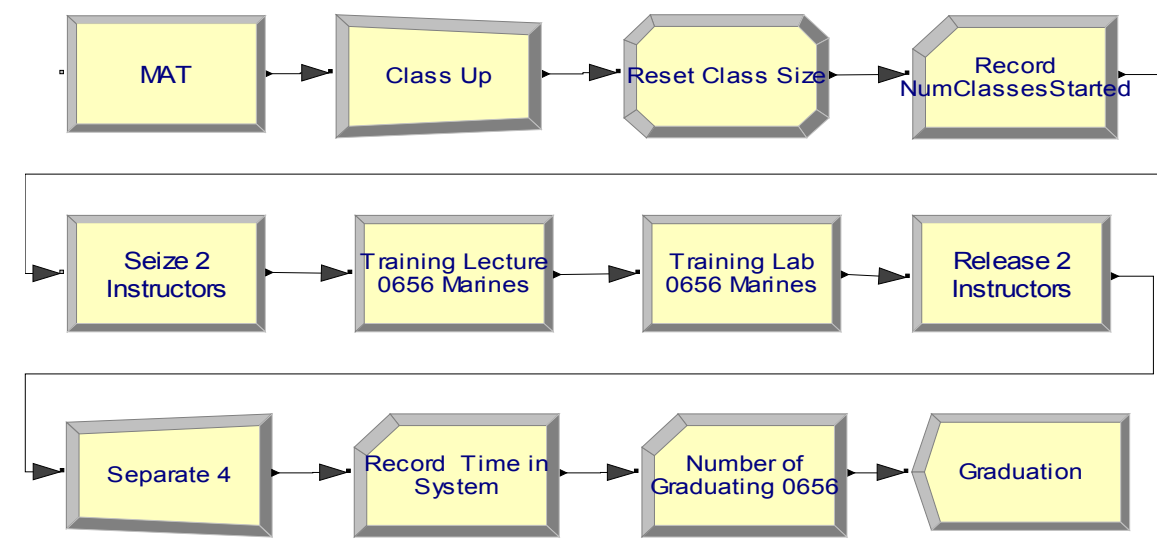

Figure 6: Example of Arena logic for simulating the training process.

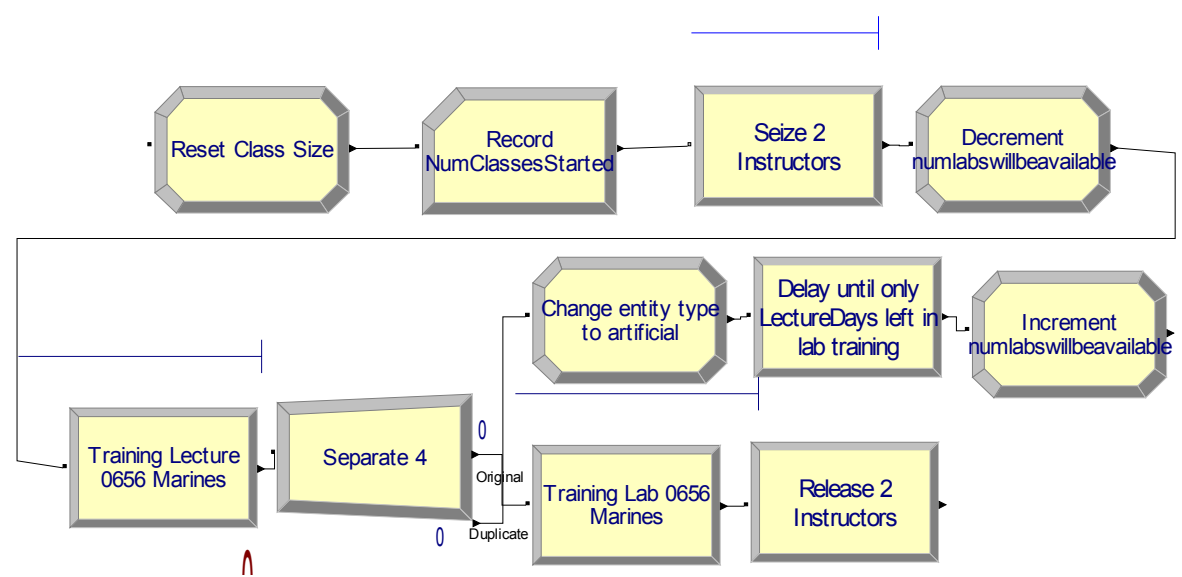

Figure 7: Example of Arena logic for decrementing and incrementing the number of lab classrooms available to allow the evaluation of whether a lab will be available before class starts are allowed

Once the class completes the lab, the Marines are then separated and counted for graduation.

\subsection{On Demand Version}

The on-demand model has six main parts with four parts being the same as the model using a schedule for class starts. Arriving students and students previously existing students in the system are created in the same manner as in the scheduled-classes model. In addition, the wait in the MAT queue and the training processes are the same.

The main difference between the models is that here classes are started immediately upon four conditions being met rather than limiting class starts to a schedule. And, in order to correctly start classes and ensure the queue remains in MAT instead of between the two processes simulating the lecture and lab classes, a variable was created "numlabswillbeavailable". This variable is changed on a counter that is decreased when a class enters the lab process and is increased when the existing class in the lab process has completed enough time in the lab that it will be available immediately for the next class to use when it finishes the lecture class process. This ensures that no class will have to wait for a lab after finishing the lecture. This is required to ensure the comparison of the models is exclusively between scheduled and on demand class starts. The actual process at MCCES schedules the class starts to transition between the lecture and lab processes without waiting. Attention to the accurate comparison of the methods of starting of classes scheduling models without changing the behavior of the processes made the on-demand model more complex to create.

While students wait in the MAT queue waiting for a class start signal, the part of the model shown in Figure 7 scans for a minimum sufficient number of students to start a class, for two available instructors, and for an available lecture classroom. In addition, an available lab is ensured by checking that the value of "numlabswillbeavailable" is greater than zero. The condition statement in the signal module is ( (NQ(MAT.Queue) $>=$ minclass) \&\& ( $\mathrm{NR}$ (Instructors) $<=$ num0656instructors-2 ) \&\& ( $\mathrm{NR}($ Lecture Halls $)<2) \& \&$ ( numlabswillbeavailable $>$ $0)$ ).

In order to evaluate the number of lab classrooms available, the logic initializes the variable of numlabsavailable equal to two and begin the system warm-up. 
The variable is decreased by one before a class enters the lecture process to guarantee a lab is reserved for that class entering the lecture process. The variable is increased after a time delay that begins after the class completes the lecture process but before it enters the lab process. The length of the delay to increase numlabsavailable is equal to the duration of the lab process minus the duration of the lecture process. Also listed as variables in the model are "Labdays" and "Lecturedays" which permit us to use the PAN feature of Arena.

\subsection{Use of Variables}

For both models, the use of variables for the instructor and classroom resources and training curriculum duration parameters permitted the authors to use a Markovian process while operating the PAN feature to run the different scenarios. This enabled the authors to evaluate the performance of the models and how the quantity of output and length of time spent in MAT queue or time in system was affected by changing the parameters.

\section{EXPERIMENTAL DESIGN AND RESULTS}

By investigating all possible combinations of the five changes described in Section 3, the authors investigated 32 scenarios. The authors ran these scenarios and analyzed the results using Arena's Process Analyzer (PAN) software package.

Examining the 32 scenarios reveals that instituting even one of the changes explored would decrease MAT waiting time by at least 37 percent. This 'worst' single change would be to change to a compact training schedule of 32 training days.

The single change that produces the best results in terms of MAT queue reduction is going to an on-demand scheduling routine. This change results in a reduction from an average 36.01 days in queue to and average of 6.33 days, representing a 82.4 percent savings in days spent waiting. Further, the average number of Marines waiting decreases from 40.84 to 6.93 . Interestingly, instructor utilization increases slightly from 83.7 percent to 86.4 percent.

The easiest single change that MCCES could immediately implement would be to increase the number of Marines allowed per computer thus increasing classroom capacity to 27 Marines. This yields a 54 percent reduction in waiting time.

The next easiest change that MCCES could implement in isolation would be to increase instructors from 10 to 12 by shifting resources from another process at MCCES. By adding two additional instructors, a 48 percent reduction in waiting time occurs.

\section{CONCLUSIONS}

By modeling the training system, the authors have shown that even making the easiest single proposed change to the training system has the potential to reduce average waiting time in MAT by 54 percent.

Changing from scheduled classes to on-demand classes would require coordination throughout the Marine Corps' various organizations involved in recruiting and training Marines. Therefore, the ability of the local commander to implement this change is limited.

The next best option for MAT queue reduction within the purview of the local commander at MCCES is to change the maximum number of students in class from 18 to 27 thereby reducing the average queue time from 36.01 days to 16.52 days, a 54.1 percent reduction in waiting. The average number waiting decreases from 40.84 to 18.18. Average instructor utilization also decreases from 83.7 percent to 63 percent.

In tandem with the change in maximum number of students per class, the next best option for MCCES' commander would be to add two instructors. This change in conjunction with the previous yields a average reduction in MAT queue waiting length of 12.2 percent from 16.52 to 14.51 days. The average number of Marines waiting also decreases from 18.18 to 15.88 , a reduction of 12.63 percent. Average instructor utilization is 53.7 percent.

These two changes represent the best the local commander can unilaterally implement. Overall, from the current operations, the savings potential is a decrease in waiting time by 59.72 percent, a reduction of the average number of Marines in MAT by 61.10 percent, and 35.84 percent reduction in instructor utilization.

Quantifying the time saved in MAT in terms of dollars can be looked at from many different angles in terms of daily salaries of Marines waiting, to costs in facilities maintenance, energy consumption, and support requirement costs, and is beyond the scope of this paper. Moreover, each day a Marine spends in MAT represents an opportunity cost to the Marine Corps. Lost days in MAT an contribute to decreased operational readiness and wasted resources.

There are other changes that could be explored to assess further impacts on waiting times. For example, future models will quantify the effects of incorporating additional lecture and laboratory facilities, further reduction in the number of required training days, increasing available instructors, and a combination scheduled versus ondemand classes.

During the analysis of the data, it was noted that the inter-arrival rates for Marines appears to be seasonal and that the lecture and lab classes are scheduled as one process without a wait between portions of the training curriculum. Future iterations of the model will incorporate 
Davenport, Neu, Smith, and Heath

Table 1: Scenarios with results. Bold indicates the results of the current system.

\begin{tabular}{|c|c|c|c|c|c|}
\hline Scenario & $\begin{array}{c}\text { Min } \\
\text { Class } \\
\text { Size }\end{array}$ & $\begin{array}{c}\text { Max } \\
\text { Class } \\
\text { Size }\end{array}$ & $\begin{array}{c}\text { Avg } \\
\text { Days } \\
\text { in } \\
\text { MAT } \\
\text { Queue }\end{array}$ & $\begin{array}{c}\text { Avg } \\
\# \\
\text { in } \\
\text { MAT } \\
\text { Queue }\end{array}$ & $\begin{array}{l}\text { Avg } \\
\text { Inst } \\
\text { Util }\end{array}$ \\
\hline OD - 32d - 10inst & 15 & 27 & 4.35 & 4.70 & 0.57 \\
\hline $\mathrm{OD}-32 \mathrm{~d}-12 \mathrm{inst}$ & 15 & 27 & 4.35 & 4.70 & 0.48 \\
\hline OD - 41d - 10inst & 15 & 27 & 4.49 & 4.86 & 0.73 \\
\hline OD - 41d - 12inst & 15 & 27 & 4.49 & 4.86 & 0.60 \\
\hline $\mathrm{OD}-32 \mathrm{~d}-12 \mathrm{inst}$ & 15 & 18 & 5.74 & 6.24 & 0.57 \\
\hline OD - 32d - 10inst & 15 & 18 & 5.74 & 6.24 & 0.68 \\
\hline OD - 32d - 10inst & 18 & 27 & 5.87 & 6.35 & 0.52 \\
\hline $\mathrm{OD}-32 \mathrm{~d}-12 \mathrm{inst}$ & 18 & 27 & 5.87 & 6.35 & 0.43 \\
\hline OD - 41d - 10inst & 18 & 27 & 5.93 & 6.43 & 0.66 \\
\hline OD $-41 d-12$ inst & 18 & 27 & 5.93 & 6.43 & 0.55 \\
\hline OD - 41d - 12inst & 15 & 18 & 6.31 & 6.91 & 0.72 \\
\hline OD - 41d - 10inst & 15 & 18 & 6.33 & 6.93 & 0.86 \\
\hline OD - 32d - 10inst & 18 & 18 & 8.00 & 8.69 & 0.66 \\
\hline $\mathrm{OD}-32 \mathrm{~d}-12 \mathrm{inst}$ & 18 & 18 & 8.00 & 8.69 & 0.55 \\
\hline OD - 41d - 12inst & 18 & 18 & 8.52 & 9.30 & 0.70 \\
\hline OD - 41d - 10inst & 18 & 18 & 8.53 & 9.31 & 0.84 \\
\hline Sch $-32 d-12$ inst & 15 & 27 & 14.05 & 15.37 & 0.44 \\
\hline Sch $-41 d-12$ inst & 15 & 27 & 14.51 & 15.88 & 0.54 \\
\hline Sch $-32 d-10$ inst & 15 & 27 & 15.27 & 16.74 & 0.52 \\
\hline Sch $-32 d-12$ inst & 18 & 27 & 15.72 & 17.15 & 0.42 \\
\hline Sch - 41d - 12inst & 18 & 27 & 16.27 & 17.78 & 0.51 \\
\hline Sch $-32 d-12$ inst & 15 & 18 & 16.38 & 17.92 & 0.57 \\
\hline Sch - 41d-10inst & 15 & 27 & 16.52 & 18.18 & 0.63 \\
\hline Sch $-32 d-10$ inst & 18 & 27 & 17.01 & 18.60 & 0.50 \\
\hline Sch - 41d - 10inst & 18 & 27 & 18.26 & 20.04 & 0.61 \\
\hline Sch $-41 d-12$ inst & 15 & 18 & 18.56 & 20.44 & 0.71 \\
\hline Sch $-32 d-12$ inst & 18 & 18 & 18.81 & 20.57 & 0.56 \\
\hline Sch $-41 d-12$ inst & 18 & 18 & 21.02 & 23.10 & 0.71 \\
\hline Sch - 32d - 10inst & 15 & 18 & 22.61 & 25.06 & 0.68 \\
\hline Sch $-32 d-10$ inst & 18 & 18 & 25.15 & 27.84 & 0.67 \\
\hline Sch - 41d - 10inst & 15 & 18 & 36.01 & 40.84 & 0.84 \\
\hline Sch - 41d - 10inst & 18 & 18 & 38.59 & 43.63 & 0.83 \\
\hline
\end{tabular}

seasonality and a class scheduling philosophy of scheduling lecture and lab classes individually since the lab classroom has been identified as a capacity limiting resource.

Lastly, future models will use more historical data provided by MCCES to develop forecasting models that, when used in tandem with the simulation model, will allow MCCES to better predict inter-arrival rates, determine the best use of its resources or justify additional changes to the system.

\section{REFERENCES}

Kelton, W. D., R. P. Sadowski, and D. T. Sturrock. 2007. Simulation with Arena. 4th ed. Upper Saddle River, New Jersey: Prentice-Hall, Inc.

\section{AUTHOR BIOGRAPHIES}

JON DAVENPORT is a Major in the United States Marine Corps. He is currently attending the Naval Postgraduate school in the MBA degree program with a concentration in logistics. His military experience includes various positions within ground logistics. His email address is $\langle j w d a v e n p e n p s$. edu $>$.

CHARLES NEU is a Lieutenant Commander in the United States Navy. He is currently attending the Naval Postgraduate school in the MBA degree program with a concentration in logistics. His military experience includes various positions within logistics and surface warfare. His email address is $<$ crneu@nps . edu $>$. 
WILLIAM SMITH is a Captain in the United States Marine Corps. He is currently attending the Naval Postgraduate school in the MBA degree program with a concentration in logistics. His military experience includes various positions within the communications field. His last assignment was as the Executive Officer of Bravo Company, MCCES. His email address is <wrsmithenps.edu>.

SUSAN K. HEATH is an assistant professor of logistics and operations management in the Graduate School of Business and Public Policy at the Naval Postgraduate School. She received her Ph.D. in management science and information systems from the University of Texas at Austin in 2006. She also has a Master of Engineering degree in operations research and industrial engineering and a Bachelor of Science in psychology, both from Cornell University. From 1997 to 2000 she worked as a senior business analyst in information systems for Kraft Foods, Inc. She has published in Interfaces, has a forthcoming publication in the European Journal of Operational Research, and has been a guest speaker at several conferences and universities. Her email address is <skheathenps.edu>. 